\title{
RESEÑ $A$ DE LIBROS
}

Angel Fucaraccio y otros, Imperialismo y control de la población, Buenos Aires, Editorial Periferia, 1973, 199 pp.

Con frecuencia sucede que la recopilación de artículos escritos con arreglo a fines determinados y diversos llega a coincidir en muy poco con los fines de la propia recopilación, a pesar de que ésta se haya preocupado por reconocer en aquellos los rasgos que les eran comunes.

Tal vez éste sea el caso de la obra que reseñamos ya que, considerados independientemente, los artículos que la integran habían aparecido hace algunos años y su contenido obedecía a múltiples preocupaciones, que iban desde la mera reflexión suscitada por la problemática demográfica y económica general (Ovsienko), hasta la denuncia severa de las prácticas de control de nacimientos realizadas por instituciones tanto oficiales y privadas como nacionales y extranjeras (Slutzky y NACLA), pasando por la refutación y la crítica de determinadas tesis y determinadas creencias acerca del crecimiento de la población y el desarrollo económico (Singer y Fucaraccio).

Sin embargo, no por ello puede decirse que la reunión de estos artículos sea del todo injustificada, ya que en su lectura siempre es posible percibir una misma actitud que orienta la argumentación: dar una respuesta al 1lamado problema del crecimiento de la población en los países en desarrollo, hasta ahora mayormente atendido e interpretado por la investigación oficial.

En términos generales, puede decirse que los autores ubican tal respuesta de frente a la intención imperialista de influir en las decisiones que los gobiernos nacionales toman respecto a las modificaciones de la población, así como de reducir estas políticas de población a programas de control de nacimientos, tanto en su formulación como en su puesta en práctica. Para ello se presentan datos e informaciones que, por su significancia, enfatizan y hacen contundentes algunas conclusiones que se derivan de su interpretación: nombres y actividades de instituciones de planificación familiar, de personajes ligados a una o más de ellas, presupuestos empleados en programas controlistas, estudios en proceso que harían más efectivos tales programas, etc., entre otras.

Así pues, casi todos los autores plantean desde el principio, obviando frases sutíles y acusaciones veladas, la existencia de programas que tienen como objetivo inmediato el control de los nacimientos para lograr, a través de esta medida, la regulación del desarrollo de la población de acuerdo a cifras bajas de crecimiento. Una vez unos y otra vez otros, van descubriendo a un Malthus resuscitado en las palabras de McNamara y en las argumentaciones de Coale y Hoover, mismas que serían la expresión de aquella ideología que pretende conservar los privilegios obtenidos por determinados países económicamente "atrasados", manipulando para ello las implicaciones derivadas del crecimiento de la población.

Entre los señalamientos más importantes que los autores hacen a la corriente neomalthusiana en general y a algunos de sus representantes en 
particular, se cuentan los siguientes: 1) el evidente carácter de clase de toda política de población que sea conducida, sin más, a la disminución del estado actual de la fecundidad en los países del Tercer Mundo; se insiste aquí en que son las clases dominantes las que financían la formulación y la realización de tales políticas, y en que éstas van dirigidas hacia las clases más bajas de la sociedad por cuanto que, de todas las clases sociales, son éstas las que presentan los niveles de fecundidad más elevados, además de constituir mayoría en la cifra de población; 2) Los modelos económicos que generalmente se utilizan en el análisis de la relación entre el crecimiento de la población y el desarrollo económico, no corresponden a las realidades de los países a los que se aplican y, por ende, no pueden aproximarse a la explicación y conocimiento de dicha relación, de suyo compleja; no existe, pues, una "ley" que pueda dar cuenta de la relación entre la tasa de crecimiento de la población y algunos indicadores del desarrolo económico: "Es sabido que todos los estados socialistas... desarrollan su economía con ritmos significativamente más rápidos que otros países. Y, además, esto no se verifica en absoluto a costa de la disminución de la natalidad", como afirma Ovsienko (p. 45) ; 3) Una de las implicaciones más importantes y, tal vez por lo mismo, más expresamente ocultada, es aquella en la que el crecimiento de la población, llevado a cifras bajas, produciría o facilitaría el desarrollo económico deseado. El sistema educativo, los medios de empleo, los recursos naturales, etc., podrían de esta manera adecuarse a las necesidades sociales correspondientes. A esta falacia responden los autores con el argumento opuesto: un mejor sistema educativo, una mayor capacidad del sistema económico para absorber fuerza de trabajo, una tecnología capaz de multiplicar la capacidad productiva de alimentos, etc., facilitarían, de ser socialmente conveniente, la posterior disminución de la fecundidad general, ya que todo ello pone al individuo en mejor posición de racionalizar su comportamiento reproductivo.

Todas estas observaciones son, a todas luces, justas, ya que son el resultado de un análisis comprometido y serio. Sin embargo, casi todos los argumentos presentados hacen referencia a uno sólo de los aspectos de la problemática a la que atienden, y es el aspecto ideológico, sobre el que, por cierto, no han sido capaces de remontarse en atención al otro aspecto, el científico, refutando planteamientos, pero casi nunca presentando alternativas. ¿Cómo estudiar, pues, la población y sus relaciones, dado que los estudios bajo crítica no lo han logrado?, ¿qué relación hay, entonces, entre la acumulación de capital y la cifra de población obrera?, ¿cuál entre el censo obrero y el censo total?, etc., son preguntas que sugiere la lectura pero que no encuentran respuesta clara en los artículos reunidos en el libro. Y esto tal vez se deba a una razón que nos parece fundamental, y que tiene que ver con la "concepción del mundo" que parece advertirse en los autores. No se trata, rigurosamente hablando, de un marxismo consecuente, sino de un marxismo del que se toman ciertos principios básicos y se desecha el resto, es decir, de un marxismo que, lejos de ser ortodoxo en el método, sólo lo es en la denuncia, de un marxismo que dramatiza al grado de vincular íntimamente el ritmo de crecimiento con las condiciones objetivas que deben existir en el inicio de un proceso revolucionario (que sería por lo cual tendrían sentido y significancia los programas de control natal), de un marxismo mistificado, en una palabra: de un marxismo ajeno a Marx. 
Por lo demás, creemos que en una reseña de artículos reunidos en forma de libro es fácil descuidarse y dejar de lado aspectos importantes de la obra; que se corre el peligro de perder las singularidades a nombre de su consideración en conjunto y que, por ende, es posible llegar a apreciaciones con las que no necesariamente estarían de acuerdo todos los autores, sobre todo porque la problemática en cuestión da lugar al debate ideológico y científico. Precisamente por ello es recomendable la lectura de este libro.

\section{Abelardo Hernández Millán El Colegio de México}

Saúl Trejo Reyes, Industrialización y empleo en México, México, Fondo de Cultura Económica, 1973, 198 pp.

El objetivo fundamental de este trabajo, nos dice su autor en la introducción, es estudiar la relación que ha existido entre el crecimiento de la producción del sector industrial y la expansión de las oportunidades de empleo en el México de la postguerra. Se dice que, con igual propósito, se analizarán también las consecuencias del desarrollo industrial en la distribución del ingreso. Ambos aspectos representan sin duda objetivos de investigación especialmente ambiciosos, y acerca de los cuales difícilmente puede afirmarse que se haya dicho la última palabra.

El autor destaca el problema de la escasez de información como una de las limitaciones para integrar estudios sectoriales en el análisis global de aspectos sociopolíticos y económicos en México, y confía en que este trabajo auxilie en la elaboración de estudios agregados del proceso de desarrollo económico, social y político de México.

Uno de los argumentos centrales de este libro es que el desarrollo industrial afecta la distribución del ingreso, por la vía de su capacidad de generación de empleos a diferentes niveles de salarios. Se afirma que nos "interesa la distribución del ingreso por la estrecha relación que guarda con el empleo industrial, pues la cantidad de plazas generadas... es uno de los factores clave para determinar cómo se distribuye el ingreso personal entre los distintos estratos de la población" (p. 12). A lo anterior se agrega la consideración de que el reparto de la riqueza afecta la estructura política del país. Se dice que el grado de democratización de un sistema político no puede desligarse del nivel de bienestar económico de la mayoría de los miembros. A este respecto surge la pregunta sobre la dirección causal de los fenómenos: ¿tiene la distribución del ingreso un impacto importante en las características institucionales del sistema político y en la distribución del poder entre los grupos sociales, o es a la inversa?

Se critica el hecho de que en México durante los últimos veinticinco años el grueso de la inversión se ha dirigido a la industria y otras actividades modernas de la economía, a pesar de que éstas generan una menor cantidad de empleo que otras actividades alternativas en las que pudo haberse invertido la misma cantidad de recursos. (No se precisa cuáles podian haber sido estas actividades.)

Coincidimos con la crítica sobre la excesiva concentración de las inversiones en determinados sectores. Sin embargo, en cierta forma desconcierta 
al lector la opinión de que, por un lado, las actividades modernas "son las que generan una menor cantidad de empleo"; y por el otro se hace el reconocimiento en páginas posteriores de que el sector moderno dentro de la industria ha sido el que ha generado la mayor proporción de empleos (pp. 127, 136 y nota de pie, p. 130). El hecho de que las empresas grandes o modernas tengan un mayor indice de productividad por hombre ocupado -o sea que, utilicen menor cantidad de mano de obra por unidad de producto no es por si mismo evidencia de su menor capacidad de generación de empleos.

La presentación del libro se hace en siete capítulos. El primero se dedica a examinar el desarrollo de la economía mexicana a partir de 1940. Se describen en forma detallada las características del proceso de industrialización, los efectos del proteccionismo en el proceso de sustitución de importaciones y la contribución de la política gubernamental a este proceso. En la p. 35 se dice que "el desarrollo del sector manufacturero en cuanto a la creación de empleos puede considerarse satisfactorio en relación a lo observado en otros países", aunque dada la elevada tasa de crecimiento demográfico y el gran número de desempleados y subempleados en la agricultura y servicios es necesario mejorar nuestro conocimiento de las fuerzas que determinan el crecimiento del empleo en los sectores altamente productivos. La consideración anterior es acertada y enfatiza la importancia de emprender investigaciones posteriores sobre las causas del desempleo y subempleo de la mano de obra en otros sectores aparte del manufacturero.

En el capítulo II se analizan varios factores que, se considera, afectan el incremento del empleo. Dichos factores son: 1) la sustitución de importaciones, 2) los cambios en la estructura del valor agregado, 3) los incrementos en la productividad y 4) los cambios en la importancia relativa de las empresas "modernas" y "tradicionales". Con excepción del efecto de la sustitución de importaciones, al cuantificar el impacto de los demás factores se encuentra que todos han tenido un papel negativo en los niveles de empleo. El calificativo de "negativo" tiene un significado relativo, y el autor mismo lo reconoce. Dados los supuestos y características de la metodología usada, los niveles de empleo hipotéticos se obtienen suponiendo que no se hubieran presentado cambios en la estructura del valor agregado, la sustitución de importaciones, los aumentos de productividad y la mayor importancia relativa de las empresas modernas, respectivamente. La obtención de un efecto "negativo" no debe extrañar si se tiene en cuenta, por un lado, el hecho de que todo proceso de desarrollo y capitalización va acompañado de aumentos de productividad media de la mano de obra $\mathrm{y}$, por otro, el supuesto subyacente de que una mayor productividad de la mano de obra equivale a un menor incremento de los niveles de empleo. Igualmente una mayor preponderancia de las industrias y empresas con mayor productividad por hombre ocupado inevitablemente se reflejará estadísticamente en un desplazamiento relativo de mano de obra.

En vista de la gran importancia (como efecto negativo) que se concede al crecimiento de la productividad de la mano de obra sobre la generación de empleo, se dedica el capítulo III a examinar los distintos factores que, se supone, influyen en el primero de estos conceptos. Entre las variables que podría decirse influyen en la producción por trabajador están: 1) la relación de salarios a precios del capital $(w / r)$, la cual influye 
en la elección tecnológica y la relación capital mano de obra que la empresa desea utilizar; 2) por las tecnologías disponibles a través del tiempo y 3) las economías de escala que aparecen al aumentar el tamaño de las empresas. $O$ sea, se supone que influyen tanto aspectos denominados "de mercado", como "tecnológicos" en la productividad de la mano de obra. La inclusión de las variables 1) y 2) mencionadas arriba obedece a consideraciones hipotéticas. La idea subyacente es que la variación de los precios relativos de los factores afecta la elección tecnológica. Sin cmbargo, vale la pena señalar que la existencia de tal conexión hasta ahora no ha sido confirmada (ni rechazada) de manera irrefutable. Por lo tanto, toda argumentación que tienda a explicar la creciente intensidad de capital en virtud de cambios en los precios relativos favorables al capital tiene una validez muy relativa. Una de las mayores dificultades para probar la validez de esta hipótesis es la disponibilidad de información idónea. Los datos existentes sobre las tendencias de la relación global nacional entre salarios y precio de unidad del capital no permite generalizar sobre su impacto a nivel de empresas de distintos tamaños.

En los capítulos IV y $\mathrm{V}$ se presenta un examen de las diferencias entre empresas de distintos tamaños, y sus diferentes tasas de expansión, respectivamente. Para esto se estiman funciones de producción en regresiones de corte transversal para empresas de distintos tamaños en una misma industria. Se concluye que hay diferencias tecnológicas y financieras importantes entre empresas de distinto tamaño en una misma industria; que las economías de escala no son de gran importancia para explicar la ventaja de las grandes empresas sobre las pequeñas, y que las empresas de distintos tamaños no se encuentran en la misma función de producción, es decir, que el análisis tradicional de la empresa debe ser modificado, para tomar consideración del carácter dual del sector manufacturero en México (p. 117). En el captíulo $V$ se examina el aumento en la importancia relativa de las empresas modernas y su efecto en el empleo.

El capítulo VI es especialmente interesante, pues trata de relacionar "el efecto del crecimiento del empleo industrial sobre la distribución del ingreso". El autor subraya la importancia de distinguir entre "participación pagada al trabajo" y "participación total del trabajo" (que incluiría los ingresos de los propietarios-gerentes de las empresas más pequeñas, el cual no se capta en los censos industriales). Para el análisis existió el problema de la disponibilidad de datos; ya que solamente el censo de 1965 contiene la información requerida para empresas por tamaño. Estadísticamente se observa que la participación de los salarios pagados en el valor agregado aumenta a medida que es mayor el tamaño de las empresas (cuadro 38). Con el fin de incorporar en el análisis el concepto de "participación total del trabajo", el autor hace el supuesto de que los ingresos de los propietarios-gerentes de las empresas más pequeñas (de 1 a 15 trabajadores) es $50 \%$ mayor que el salario medio del trabajador en el sector de pequeñas empresas en cada industria. En consecuencia, la participación total del trabajo en las pequeñas empresas aumenta sustancialmente (cuadro 39), y las conclusiones sobre el impacto de las mayores empresas en la distribución del ingreso son diametralmente opuestas a las que podríamos derivar al considerar sólo la participación pagada del trabajo. Es evidente en este caso la importancia de los supuestos en el tipo de conclusiones que se extraen del análisis. 
Este capítulo es interesante y sugestivo, pues no obstante las limitaciones ce información, se hacen alcances y conjeturas para explicar las tendencias en la distribución del ingreso. El autor destaca el hecho de que al parecer "el desplazamiento relativo de las empresas pequeñas por las grandes en cada industria, ha tenido un efecto positivo en la participación pagada de la mano de obra en el valor agregado". Además, la posibilidad de mejorar "la distribución personal del ingreso depende de manera muy importante de la cantidad de empleos adicionales que se generen como resultado de los incrementos en la producción del sector moderno" (p. 156). Esta es una conclusión de indiscutible importancia y permite al autor afirmar que si el empleo resultante de los incrementos en la producción (del sector moderno) no es suficiente para absorber a las personas de nuevo ingreso a la fuerza de trabajo urbana, se incrementará la desigualdad absoluta y relativa de salarios dentro del sector obrero; al no generarse suficientes oportunidades de empleo productivo en el sector industrial y en otras actividades, el resto de la población no podrá participar de los beneficios del desarrollo.

Finalmente, en el capítulo VII, se considera el problema de la ocupación y las perspectivas de creación de empleos en el sector industrial. Se menciona que el verdadero problema a nivel de toda la economia es la subocupación, y no el desempleo abierto. Se subraya que el panorama no es halagüeño. Dado que para 1980 la fuerza de trabajo será de aproximadamente 21 millones, para eliminar la subocupación será necesario aumentar en 14 millones el número de empleos de alta productividad durante el decenio de los setenta.

Uno de los méritos principales de la obra que se comenta es el señalamiento de la gran importancia del problema del empleo en el futuro del país. Contiene pasajes de sumo interés y enfoques metodológicos que sin duda estimularán investigaciones posteriores. Debe además reconocerse la gran escasez de investigaciones empíricas que consideren conjuntamente las tendencias del empleo y la industrialización en México. Su lectura, por lo tanto, es altamente recomendable para los interesados en el análisis empírico de estos conceptos.

Sofía Méndez VillarReal El Colegio de México

Joan Robinson y John Eatwell, Introduction to Modern Economics, Londres, McGraw Hill, 1974, 349 pp.

Esta obra, dicen los autores en el prefacio, está dirigida en primera instancia a estudiantes que se incian en economía, aunque algunas partes pueden interesarle a un público más amplio. Si aceptamos la idea de que el objetivo más importante en la enseñanza de la economía debe ser que el estudiante adquiera los elementos necesarios para comprender la forma en que operan los modernos sistemas económicos, así como que debe darse mayor énfasis al análisis que a la descripción de los procesos económicos, entonces el libro que se comenta representa una verdadera contribución a los esfuerzos por avanzar en esa dirección.

Para comprender la teoría económica moderna y para poder darle su debida dimensión, es necesario conocer en qué medida ha sido una deriva- 
ción de planteamientos iniciales o, alternativamente, una reacción en contra de enfoques precedentes. Esto implica ubicar históricamente el surgimiento y desarrollo del pensamiento y del análisis económico. Implica también tener en cuenta los aspectos institucionales en que el argumento económico se ha desarrollado. En este sentido, uno de los principales aciertos de este libro es su insistencia en que el elemento esencial en cualquier análisis económico es la consideración explícita del tipo de sistema social al que se aplica. Para los autores, es de una importancia fundamental que se especifique el sistema institucional dentro del cual se aplican, o suponemos que se aplican, nuestros razonamientos.

Aunque por el título podría pensarse que se trata de un libro de tipo elemental, no lo es. Lo introductorio no le confiere un carácter elemental. Hay algunas partes que difícilmente serán totalmente accesibles al estudiante de primer año de economía. La complejidad de estas partes es simplemente reflejo de la complicación de algunas de las controversias teóricas actuales. Lo anterior es hasta cierto punto inevitable en virtud del gran interés de los autores de enfocar los argumentos y la metodología hacia la adopción de una posición ante las modernas controversias. En alguna medida esta conjuga las más importantes proposiciones de la corriente de Cambridge (Inglaterra). Por sí mismo, el esfuerzo de los autores representa un punto más a favor de la opinión que enfatiza la importancia de considerar el desenvolvimiento de la ciencia económica a la luz de las contribuciones de Sraffa. Algunos de los aspectos que probablemente presentarán dificultades al estudiante que se inicia en economía son los referentes a las discusiones sobre la teoría de la distribución, los problemas referentes a la medición del capital, e incluso el modelo de Sraffa, del cual se hace una excelente presentación. La complejidad inevitable de algunas de éstas cuestiones no es en sí un obstáculo insalvable, y en cambio permite ubicar al estudiante dentro de las discusiones de la economía teórica moderna.

La presentación se divide en tres secciones o temas: doctrinas económicas, análisis, y problemas modernos. En la primera se hace una reseña de las principales líneas de pensamiento económico desde el siglo XVIII hasta nuestros días. En opinión de los autores, el desarrollo de la teoría de la economía política ha contribuído a la creciente autoconciencia de la opinión intelectual que siguió a la revolución científica del siglo xvirr. En la parte introductoria se hace referencia a los problemas y funciones de la filosofía económica. Los autores destacan que es fácil ver que el trabajo crea riqueza y que la naturaleza provee los frutos de la tierra, pero... hay también utilidades ¿de dónde provienen estas utilidades? ¿es que el capital crea riqueza como el trabajo? o ¿las utilidades sólo representan una carga sobre la riqueza creada por el trabajo? En esta parte se aborda también el problema de los precios los cuales se consideran la manifestación más evidente de la actividad económica. Sin embargo, sucede que varían erráticamente $y$, en consecuencia los autores se preguntan si hay algún principio de valor subyacente que explique sus fluctuaciones: y respecto al problema del dinero; ¿cuál es el papel del dinero en la economía?, ¿qué relación hay entre el ingreso monetario de los individuos y la riqueza de la sociedad en su conjunto? En el caso de que todo mundo tenga más dinero para gastar, pero la oferta de bienes permanece constante ¿quienes serían los que se benefician?

Se discute también el problema de la justicia social: ies correcto que algunas familias vivan con lujo extravagante, mientras otras apenas pueden 
conseguir alimentos para sus hijos? Esta es un cuestión difícil en verdad y a la cual, según los autores, ninguna escuela de pensamiento (incluso en la Unión Soviética) ha dado una respuesta satisfactoria. Finalmente se analiza el problema de la demanda efectiva: ¿de dónde viene la demanda y por qué rara vez es suficiente para mantener a todos en pleno empleo?

Los autores consideran también la relación entre ciencia y metafísica, siendo esta última el tipo de razonamiento que a menudo se usa en las ciencias sociales. De acuerdo con los autores, en los razonamientos de tipo metafísico el lenguaje utilizado no converge en información factual, no describe relaciones lógicas y no da instrucciones precisas. Sin embargo se utiliza para influir en el comportamiento. En economía, por ejemplo, el concepto tan atractivo de la armonía natural es metafísica pura. Igualmente aquello de que "lo mejor para el mayor número" es un concepto metafísico, en el que no se propone ningún criterio para elegir "lo bueno", ni tampoco para optar entre darle lo más "bueno" a unos pocos, o darle lo menos "bueno" a muchos.

En los tres capítulos que integran esta parte se consideran respectivamente las ideas que precedieron a Adam Smith, la economía política clásica y la neoclásica. Al analizar cada una de las doctrinas económicas los autores se esmeran por distinguir entre los elementos de hecho y de lógica de los elementos metafísicos incorporados en el análisis.

La sección dedicada al "Análisis", se compone de once capítulos y constituye la parte esencial de la obra. La importancia que los autores conceden al análisis no es sino el reflejo de sus intereses teóricos y metodológicos. En lo que se refiere al método que utilizan, explican que éste consiste en seleccionar de la historia (incluyendo el presente como historia), entidades tales como bienes, precios y unidades monetarias, tierra cultivable, equipo productivo, patrones, trabajadores y propietarios de riqueza, e integrar estas entidades dentro de un modelo en el cual las interacciones se establecen a través de una lógica semimatemática. El modelo representa a la vez una hipótesis y una simplificación de la realidad. El arte de elaborar modelos consiste en descartar las complicaciones innecesarias para los objetivos planteados, sin eliminar las características útiles y relevantes. En su opinión, el elemento esencial en cualquier análisis es el tipo social al que se aplica. Consideran además las relaciones económicas (entre individuos) y las relaciones técnicas (entre individuos y el universo físico) y afirman que las relaciones entre los individuos de una sociedad tienen una influencia muy importante en el tipo de tecnología que se desarrolla, de modo que las relaciones entre individuos y las relaciones tecnológicas constituyen la materia central de estudio en el análisis económico. Los autores advierten además al lector que el argumento presentado está en términos post-Keynesianos.

La última parte, "Problemas modernos", consiste en una breve e interesante reseña de los principales obstáculos y problemas que enfrentan los países capitalistas avanzados, los socialistas y los que integran el llamado tercer mundo. En opinión de los autores, el objetivo del "análisis" no puede ser el de proponer soluciones sino sugerir al lector los aspectos a incluir para lograr una comprensión adecuada de los problemas. E1 razonamiento económico por sí mismo no puede ofrecer una solución a los problemas económicos, debido a que éstos involucran aspectos políticos, sociales y humanos dificilmente reducibles al cálculo "de más o de menos". Casi no hay 
problemas en economía cuya solución implique "hacer el bien por lo menos a uno sin perjudicar a ningún otro". Postulados de la economía del bienestar como el anterior carecen de toda relevancia práctica.

A lo largo del texto los autores hacen esfuerzos deliberados por diferenciar su obra de otros libros de texto introductorios. Uno de sus objetivos primordiales es presentar un enfoque fresco y original. En su opinión, el desarrollo de la ciencia económica adolece de serias deficiencias. Al final del libro sugieren que "es tiempo de regresar y empezar por el principio". Aunque los autores cuestionan la utilidad de algunos conceptos y corrientes de pensamiento para ayudar a responder las cuestiones esenciales de la economía política, su crítica no es destructiva y mantiene siempre la seriedad formal de un buen análisis académico. Está es una ventaja sumamente importante en un libro introductorio, pues existe el peligro de que el cuestionamiento prematuro del cuerpo teórico que se va a estudiar contribuya a desalentar al estudiante, y a menguar la seriedad con que debe emprender el estudio de la economía.

En el caso de la obra que se comenta la condición genuinamente académica de los autores y su profundo conocimiento de la materia les permite, con toda autoridad, calibrar el alcance y las limitaciones de distintos enfoques teóricos. Para ellos no se trata de rechazar a priori ninguna línea de pensamiento, pero sí de darle su debida dimensión y encuadrarla dentro del marco institucional que les corresponda. Es difícil exagerar la excelencia de esta obra, así como también es difícil preveer el impacto que tendrá en la enseñanza de la economía. Lo previsible es que tendrá una influencia tal, que su lectura difícilmente podrá ser ignorada por los economistas, incluso los más convencionales.

\section{Sofía MÉndez Villarreal El Colegio de México}

Irma Adelman y Cynthia Taft Morris, Economic Growth and Social Equity in Developing Countries, California, Stanford University Press, 1973, 257 pp.

A partir del criterio de que "la razón última del desarrollo económico es promover el bienestar humano, de manera principal a través del aumento de la participación política de los menos poderosos y del ingreso de los pobres", en este libro las bien conocidas autoras señalan que los programas convencionales de desarrollo de los últimos dos decenios, a pesar de que han logrado incrementos sustanciales en la tasa de desarrollo, no han contribuido a aliviar la miseria humana.

La idea generalizada de que los incrementos sostenidos en la tasa de crecimiento de algunas componentes del desarrollo, tales como la industrialización, la productividad agrícola, la disponibilidad de capital físico y el producto bruto per capita, están estrechamente asociados con una creciente participación política y económica es refutada en este trabajo. Las autoras encuentran que más bien el crecimiento económico no sólo va acompañado de una disminución real de la participación política sino que es una de las causas principales de la desigualdad del ingreso, por lo que, desde el punto de vista del mejoramiento de la distribución del ingreso, se concluye que 
las estrategias del desarrollo deben ser reconsideradas. Sin embargo, al igual que las autoras, cabe preguntarse cómo.

Este estudio constituye un esfuerzo preliminar por responder a esta pregunta. A través del examen cuantitativo de diversas variables utilizadas para representar un amplio número de influencias de carácter político, económico y social que a priori podría esperarse afectan la participación política y la distribución del ingreso en países en desarrollo, tratan de valorar la importancia relativa de cada una de ellas en la formulación de políticas de desarrollo factibles.

Para explicar las complejas relaciones entre participación política y económica, analizan 48 indicadores cuantitativos sobre características sociales, económicas y políticas en una muestra de 74 países no socialistas - para un periodo que abarca los años de 1957 a 1962 - cuya descripción y justificación se discute en los primeros dos capítulos. ${ }^{1}$

En el estudio de las fuerzas que tienden a incrementar la participación política en los países considerados, utilizan la técnica del análisis discriminante mediante la cual obtienen una clasificación de países en función del nivel de participación política, para analizar luego, mediante el mismo procedimiento, las relaciones más significativas que se observan entre las variables utilizadas. (capítulos 3 y 4). Después de analizar las influencias que contribuyeron al fracaso de las viejas políticas de desarrollo para lograr resultados, se sugiere la componente principal de estrategias de desarrollo alternativas para países de niveles diferentes de desarrollo.

Posteriormente (capítulo 5 y parte del 4) analizaron los resultados derivados del análisis discriminante, entre los cuales cabe señalar los siguientes:

1. Las tasas de crecimiento económico en el plazo corto no están asociadas de manera significativa al producto nacional bruto per capita y la participación política (especialmente en los países de bajo nivel socioeconómico de la muestra). ${ }^{2}$

2. La desigualdad del ingreso tiende a ser mayor cuando la explotación de recursos naturales abundantes coincide con una concentración de capital en manos de extranjeros o refugiados; y tiende a ser menor cuando las estrategias del desarrollo conceden atención preferente a las inversiones en recursos humanos, a la diversificación de las exportaciones de manufacturas y a la expansión de la producción e inversión del sector público.

3. Una vez que se alcanza cierto nivel mínimo de desarrollo, el mejoramiento de las instituciones gubernamentales, acompañado de avances sociales o de cambios en la estructura del comercio hacia una mayor diversificación en las exportaciones de manufacturas, apoyadas por políticas gubernamentales, favorece una mayor participación en el ingreso total de los grupos de ingreso medio de la población.

4. El análisis respalda el punto de vista marxista de que la estructura económica y no el nivel del ingreso o la tasa de crecimiento económico es el determinante básico del patrón de distribución del ingreso.

1 La información que utilizan proviene de otro estudio de las mismas autoras: Society, Politics and Economic Development, Baltimore, 1971.

2 Estos resultados son consistentes con la hipótesis adelantada por Kuznetz y Myrdal a principios de los años cincuenta, en el sentido de que la participación relativa de los estratos pobres de la población tiende a disminuir. 
Con base en los resultados del análisis y a partir de dos juicios valorativos acerca de las estrategias de desarrollo (que la meta principal de las políticas de desarrollo y planificación debe ser la de garantizar justicia social para los necesitados; y que cualquier patrón de desarrollo económico es injusto cuando no cumple el propósito de mejorar el nivel de vida de la mayor parte de la población) señalan algunos instrumentos para mejorar la distribución del ingreso:

a) A bajos niveles de desarrollo, las medidas para reducir el grado de dualismo económico resulta de gran importancia para mejorar la igualdad del ingreso.

b) En los países con niveles intermedios y altos de desarrollo, las estrategias tendientes a mejorar los recursos humanos y expandir el tamaño y la participación del sector público en la inversión neta (aunque esto no mejora la posición relativa de los pobres); la diversificación de las exportaciones de manufacturas; y el mejoramiento del sistema impositivo, favorecen una mayor igualdad del ingreso.

Afirman las autoras que hasta ahora, las políticas de desarrollo, que en principio parecian haber sido diseñadas para mejorar la distribución del ingreso, en realidad sólo han servido como instrumentos adicionales para incrementar la riqueza y poder de las élites existentes y, lo que es aún más serio, las cuales a menudo obtienen su poder de los programas de desarrollo y manipulan las instituciones económicas y políticas para servir a sus fines privados.

Al final, concluyen que los únicos instrumentos de política que ofrecen alguna esperanza de mejoras significativas en el estándar de vida de los pobres requiere una reorientación radical en las estrategias del desarrollo, "la única estrategia aceptable para los decenios por venir es el desarrollo del pueblo, por el pueblo y para el pueblo. Sin nuevas instituciones y políticas diseñadas específicamente para mejorar a la totalidad de los pobres no hay posibilidad real de justicia social en el mundo de nuestro tiempo".

Aún cuando los hallazgos de esta investigación sobre los problemas de desarrollo pudieran parecer triviales (mucho de lo señalado en sus recomendaciones lo manipulan con demasiada frecuencia los políticos de los paises atrasados económicamente, por lo menos en América Latina) sin embargo, una cosa es intuirlos y otra muy distinta darles sustancia teórica y empírica.

Aparte de los hallazgos de las autoras, tanto la información como la metodología utilizada constituyen un importante aporte para el estudio del desarrollo y por el conjunto de orientaciones de estartegia que contiene, no dudamos que pueda servir como una guía de inapreciable valor tanto para los teóricos de la materia como para los encargados de diseñar la política económica de los países que como el nuestro (incluido en la muestra estudiada) enfrentan graves problemas que se manifiestan en desigualdades agudas del ingreso y en una participación política reducida.

Raúl de la PeÑa El Colegio de México 
Hassan Fathy, Architecture for the Poor, Chicago y Londres, The University of Chicago Press, 1973, 233 pp. + ilustr.

El libro se refiere fundamentalmente a los problemas de la construcción de casas habitación en el campo egipcio mediante la aplicación de técnicas constructivas autodiseñables por los habitantes de la región al través del uso de los materiales propios del lugar. El autor se plantea la solución de los problemas de la habitación popular campesina después de analizar las posibilidades reales de los países pobres para encarar la situación y una vez que considera el problema de la habitación rural como una parte de la rehabilitación regional en sus aspectos fundamentales: económico, sociológico, urbanístico.

El texto constituye una descripción o crónica de las experiencias e investigaciones profesionales del arquitecto Hassan Fathy. Después de hacer algunas consideraciones sobre la forma en que entró, desde su juventud, en contacto con la vida y la organización (y la pobreza) del campesino egipcio, el autor entra de lleno al problema técnico de la construcción de la casa campesina. En este sentido, se remite a las formas ancestrales utilizadas por los campesinos del país y a la ignorancia de los sistemas que propugnaron por la utilización de los materiales de construcción contemporáneos (acero, concreto, armado, cemento, etc.). Aduce que dichos materiales sólo son aplicables a los procedimientos contemporáneos y plantea limitaciones en su empleo.

En 1941 Fathy entra en contacto con la solución técnica aplicada en Nubia desde hace siglos por los habitantes de la región. Descubre la supervivencia de la antigua técnica de la bóveda esquifada. Más tarde, una vez en El Cairo, comienza a experimentar en base a sus anteriores investigaciones y mediante la demostración práctica de que el techado y la bóveda esquifada resultaban aplicables en la introducción moderna de un nuevo sistema constructivo. En el proyecto de Nueva Gourna se interesa por la restructuración a fondo de una gran extensión rural y describe pormenorizadamente la organización del proyecto.

Los aspectos sociológicos del proyecto, así como las ideas y conceptos arquitectónicos y urbanísticos sostenidos por Fathy, se hallan descritos en el capítulo segundo. Elementos primordiales como el carácter arquitectónico, los procesos de investigación en la toma de decisiones, el papel de la tradición, la salvaguarda de la individualidad y la utilización del adobe en los sistemas constructivos son considerados por el autor como elementos de liga entre la arquitectura vernácula y la aparición de una arquitectura actualizada. Fathy sostiene que la apariencia del edificio tienen un efecto profundo en las formas de vida de los habitantes. Víncula, pues, el aspecto social con el aspecto arquitectónico y añade algunas consideraciones socioeconómicas que se refieren a las tareas artesanales e industriales del campesino egipcio. Posteriormente describe el plan de Nueva Gourna subrayando que las características urbanísticas de los servicios públicos, el mercado, el teatro, las escuelas y los espacios propios de la vida comunal deben mantener su carácter regional. Lo mismo opinará con respecto a las partes de la casa: recámara, cocina, abastecimiento de agua, servicios de higiene, pequeño establo, etcétera. 
E1 proyecto de Gourna fue para Fathy un experimento fundamental. La construcción del pueblo indicaría líneas y orientaciones para la reestructuración del campo egipcio. Su creador plantea formas de financiamiento y de organización que permitirían felices resultados. Asimismo, se refiere a los problemas surgidos durante el desarrollo del proyecto, sobre todo a los problemas técnicos que guardan nexos con el sistema de cooperativa, con la autopreparación técnica de los operarios y con la necesidad de convencer a la población de que Gourna no era una meta en sí misma sino la posibilidad de ampliar el panorama socioeconómico de los habitantes del campo egipcio. Debe aclararse que el proyecto de Gourna fue considerado al principio como la solución a una situación única y local, que posteriormente se reflejó en un Programa Nacional para la Reconstrucción Rural. A este respecto, Fathy aporta datos de planificación que en detalle exhiben cómo desarrollar la integración multidisciplinaria.

Gourna nunca se terminó. Las obras fueron suspendidas y Fathy se refiere a ello en su libro de una manera clara. Sin embargo, al considerar que el experimento había servido de base a posteriores proyectos, plantea que funcionó perfectamente como fuente de información para elaborar nuevos planes de reestructuración regional en el campo egipcio. Para dejar claramente asentados los logros, describe las etapas de los trabajos realizados, imponiéndoles características cronológicas. La primera etapa, 1945-1946, incluyó los trabajos de diseño y de investigación, la tramitación de la obra y el análisis de las formas de abastecimiento de los materiales que podrían ser utilizados en la construcción de la pequeña ciudad. La segunda etapa, que va de 1946 a 1947 , se refiere a los procedimientos utilizados para adquirir materiales y equipo, a los pasos realizados para comenzar la construcción y a los problemas surgidos para la consecución de los planes trazados (por ejemplo, una epidemia de cólera que sembró el pánico en la región). La tercera etapa (1947-1948) analiza la situación ecológica de la región y describe cómo surgieron problemas ante el desbordamiento de las aguas pluviales y con motivo de la oposición de algunos terratenientes. Fathy asienta que tuvieron que tomarse medidas radicales para solucionar estos y otros problemas y para salvaguardar la existencia misma de los implementos y el equipo adquirido para llevar a cabo la construcción de Gourna.

Tanto el estilo ameno del texto, como el hecho de que Fathy implementa al lector con datos técnicos detallados, permiten concluir que Architecture for the Poor constituye un libro de vital importancia para familiarizarse con los problemas actuales de la habitación en el campo, tanto en sus aspectos técnicos como administrativos y organizativos. Por ejemplo, el autor afirma que el experimento de Gourna no podía de ningún modo, solucionar el sistema de cooperativas. El proyecto Gourna, afirma Fathy, acabará por realizarse en Navaroh, plan que, en palabras del autor, revolucionará el sistema de abastecimiento habitacional en todo Egipto. Estas características del libro de Fathy, así como los apéndices de la obra (que incluyen el análisis de costos, los sistemas de organización del trabajo, los cálculos de cimentación, y la fabricación del tabique), hacen de Architecture for the Poor un libro completísimo tanto para el arquitecto especializado en el problema habitacional como para el lector lego que desee penetrar en algunos problemas socioeconómicos del campo en los países subdesarrollados. Las certeras fotografías que aparecen en la edición norteamericana ilustran y complementan el texto operativa e incluso didácticamente. Una última cualidad del 
libro se refiere a la inclusión de los planos arquitectónicos y urbanísticos, los esquemas de fachadas y otros dibujos técnicos, materiales de vital importancia para entender cabalmente los conceptos y las experiencias de Hassan Fathy.

Alberto Dallal

El Colegio de México 